\title{
Vitamin D supplementation and COVID-19 disease: safety but unproven efficacy—reply to Dr Helga Rhein
}

\author{
Nicholas C. Harvey ${ }^{1,2}$ (D) Cyrus Cooper ${ }^{1,2,3} \cdot$ Zahra Raisi-Estabragh $^{4,5}$
}

Received: 11 July 2021 / Accepted: 29 July 2021 / Published online: 18 August 2021

(c) The Author(s), under exclusive licence to Springer Nature Switzerland AG 2021

To the editor:

We read with interest the letter from Dr Helga Rhein, responding to our rapid evidence review around vitamin D and COVID-19 disease [1]. Dr Rhein suggests that the "evidence for the benefits of avoiding vitamin D deficiency during the pandemic far outweighs the theoretical risk of overdose" [2]. Thus, "on the balance of probabilities", should we routinely supplement the population with higher dose vitamin D than the $400 \mathrm{IU} /$ day suggested in the UK as the (dietary) RNI [3]? We completely agree with Dr Rhein that $400 \mathrm{IU} /$ day will not effectively ameliorate overt vitamin D deficiency. If an individual presents with symptoms and signs consistent with the metabolic consequences of vitamin $\mathrm{D}$ deficiency, then clearly they should be treated appropriately with more rapid replenishment $[4,5]$. This is a fundamentally different scenario to that of population health. The UK Scientific Advisory Committee on Nutrition (SACN) recommendation is aimed at ensuring that $97.5 \%$ of the population have $25(\mathrm{OH}) \mathrm{D}>25 \mathrm{nmol} / \mathrm{l}[3]$. Whilst one can argue that the therapeutic window for vitamin D supplementation is wide and risks of overdose low, we are not convinced (see our response to the letter of Dr William Grant $[6,7]$ that this is sufficient reason to introduce population-level higher dose

Nicholas C. Harvey

nch@mrc.soton.ac.uk

1 MRC Lifecourse Epidemiology Centre, University of Southampton, Southampton, UK

2 NIHR Southampton Biomedical Research Centre, University of Southampton and University Hospital Southampton NHS Foundation Trust, Southampton, UK

3 NIHR Oxford Biomedical Research Centre, University of Oxford, Oxford, UK

4 NIHR Barts Biomedical Research Centre, William Harvey Research Institute, Queen Mary University of London, London, UK

5 Barts Heart Centre, St Bartholomew's Hospital, Barts Health NHS Trust, London, UK supplementation for prevention of COVID-19 disease when the observational evidence provides very limited support and the best conducted intervention study to date suggests no benefit [8]). Whether such an approach might be warranted for other outcomes, or to prevent metabolic bone disease in high risk demographics, are separate questions.

Acknowledgements We would like to thank the Medical Research Council (UK), National Institute for Health Research (NIHR), Wellcome Trust, Versus Arthritis, Royal Osteoporosis Society Osteoporosis and Bone Research Academy and International Osteoporosis Foundation for supporting this work. ZRE was supported by British Heart Foundation Clinical Research Training Fellowship No. FS/17/81/33318.

Funding There was no direct funding for this letter.

\section{Declarations}

Conflict of Interest NCH reports personal fees, consultancy, lecture fees and honoraria from Alliance for Better Bone Health, AMGEN, MSD, Eli Lilly, Servier, Shire, UCB, Consilient Healthcare, Kyowa Kirin and Internis Pharma, outside the submitted work. CC reports personal fees, consultancy, lecture fees and honoraria from Alliance for Better Bone Health, Amgen, Eli Lilly, GSK, Medtronic, Merck, Novartis, Pfizer, Roche, Servier, Takeda and UCB. ZRE reports no conflicts of interest.

Ethical approval There was no direct involvement of patients or participants in this letter.

Informed consent Ethics review and informed consent are not applicable.

\section{References}

1. Raisi-Estabragh Z, Martineau AR, Curtis EM et al (2021) Vitamin D and coronavirus disease 2019 (COVID-19): rapid evidence review. Aging Clin Exp Res 33:2031-2041. https://doi.org/10. 1007/s40520-021-01894-Z 
2. Rhein H (2021) Vitamin D—let common sense prevail—on the balance of probabilities. Aging Clin Exp Res. https://doi.org/10. 1007/s40520-021-01939-3

3. SACN (2016) Vitamin D and health. Scientific Advisory Committe on Nutrition, London

4. Harvey NC, Cooper C (2012) Vitamin D: some perspective please. BMJ 345:e4695

5. Aspray TJ, Bowring C, Fraser W et al (2014) National Osteoporosis Society vitamin D guideline summary. Age Ageing 43:592-595

6. Harvey NC, Cooper C, Raisi-Estabragh Z (2021) Vitamin D and COVID-19 disease: don't believe everything you read in the papers! Reply to Dr William B. Grant. Aging Clin Exp Res. https://doi.org/10.1007/s40520-021-01957-1
7. Grant WB (2021) Vitamin D and coronavirus disease 2019 (COVID-19): rapid evidence review. Aging Clin Exp Res. https:// doi.org/10.1007/s40520-021-01941-9

8. Murai IH, Fernandes AL, Sales LP et al (2021) Effect of a single high dose of vitamin D3 on hospital length of stay in patients with moderate to severe COVID-19: a randomized clinical trial. JAMA 325:1053-1060

Publisher's Note Springer Nature remains neutral with regard to jurisdictional claims in published maps and institutional affiliations. 